Research Article

\title{
A Feasible and Facile Method for the Anticounterfeiting of Down Fiber
}

\author{
Qianpeng Jin, ${ }^{1,2}$ Simeng Gao, ${ }^{2}$ and Yang $X u \mathbb{D}^{1,2}$ \\ ${ }^{1}$ Key Laboratory of Eco-Textiles, Ministry of Education, Jiangnan University, Wuxi, Jiangsu 214122, China \\ ${ }^{2}$ College of Textile Science and Engineering, Jiangnan University, Wuxi, Jiangsu 214122, China
}

Correspondence should be addressed to Yang Xu; xuyang@jiangnan.edu.cn

Received 28 June 2021; Accepted 16 August 2021; Published 10 September 2021

Academic Editor: Antonio Caggiano

Copyright ( 2021 Qianpeng Jin et al. This is an open access article distributed under the Creative Commons Attribution License, which permits unrestricted use, distribution, and reproduction in any medium, provided the original work is properly cited.

In order to prevent counterfeiting of down fiber from consumers, rare earth fluorescent materials are developed in the field of material identification and anticounterfeiting. Herein, the development of verifiable down fiber based on infrared excitationinfrared emission was described. A novel method was approached to prepare security down fiber, which involved modification of down-conversion nanoparticles (DCNPs) by sulfonic groups and self-assembly onto down fiber through electrostatic force. DCNPs were successfully prepared from ytterbium-deposited $\mathrm{NaYF}_{4}$ nanoparticles using a complexation precipitation approach, in which the trivalent ytterbium ions served as the luminescent center. Sulfonic down-conversion nanoparticles $\left(\mathrm{SO}_{3}-\mathrm{SiO}_{2} @\right.$ DCNPs) were fabricated by the hydrolysis of 3-mercaptopropyl triethoxysilane (MPTES) and next oxidation to enhance the combination of the DCNPs with down fiber. The synthesis of DCNPs and $\mathrm{SO}_{3}-\mathrm{SiO}_{2} @ \mathrm{DCNPs}$ and its pendant to down were confirmed by XRD, SEM, XPS, FT-IR, Zeta potential meter, and PL, which revealed the presence of DCNPs in the size average $86 \mathrm{~nm}$. The obtained DCNPs and security down fiber were launching an invisible red-shifted emission of $930 \sim 1080 \mathrm{~nm}$ (corresponding to the ${ }^{2} \mathrm{~F}_{5 / 2} \longrightarrow{ }^{2} \mathrm{~F}_{7 / 2}$ transitions in $\left.\mathrm{Yb}^{3+}\right)$. After washing, the infrared emission of security down fiber was evaluated and proved to be effective with fine results, which showed its potential application in the field of security.

\section{Introduction}

Counterfeit product throws a growing threat to both producers and customers, as it leads to revenue loss and market chaos [1]. Textiles are prone to be counterfeited due to their massive manufacture scale. Therefore, various security technologies have been widely used in textiles to protect consumers and producers from the fake and shoddy products $[2,3]$. Numerous methods, including quick response $(\mathrm{QR})$ codes, radio frequency identification (RFID), spectroscopy analysis, and photoluminescence materials, were emerged to identify fiber and textiles [4-6]. Conversely, counterfeiters simultaneously contributed by modern science and technology are rapidly developing among kinds of fields such as raw fiber [7]. Thus, it remains a challenge for the construction of a high security level strategy for the anticounterfeiting of conventional fibers.
Photoluminescence materials with stability and tunable optical properties are considered reliable for authenticated matter, such as organic dyes, carbon quantum dots (CDs), and lanthanide-based materials. Peng et al. had synthesized a fluorescent tetraphenylethylene derivatives and testing results indicated a potential application for anticounterfeiting. [8] Fatahi et al. prepared a fluorescent cotton fiber by dyeing with hydrophilic CDs which showed an emission peak at $450 \mathrm{~nm}$ under UV excitation, whereas the application of CDs in textile is limited by the low yield of the hydrothermal preparation. [9] By contrast, inorganic photoluminescence nanomaterials with characteristic emission, as exemplified by lanthanide doped materials, received more attention because of its properties of chemical resistance and thermostability [10]. Although visible emission facilitates the identification of counterfeit by naked eye, it exposes the protocol of tracked materials during the checking process that involves a risk for deteriorating the security level of 
anticounterfeiting [3]. Hence, lanthanide-based materials with infrared emission are gradually evolved in the application of verification [11-13].

The common strategy to pick up fluorescent filaments is utilizing the mixes of photoluminescence materials and polymers to turn, for occasion, polyester, polypropylene, regenerated cellulose, and like that [14-17], yet that is inappropriate to raw natural fiber $[14,18]$. On the other hand, the complicated and high-cost preparation of several fantastic photoluminescence materials hinders its pragmatic application in textiles. In this work, a novel approach for nature down fiber anticounterfeiting was reported through electrostatic force between sulfonic down-conversion nanoparticles $\left(\mathrm{SO}_{3}-\mathrm{SiO}_{2} @ \mathrm{DCNPs}\right)$ and down, thus to endow the down with a near infrared emission while illuminating by a shorter wavelength infrared light. Notably, the combination drive between down and modified nanoparticles was inspired by protein fiber coloring, which is an adhesivefree process. The $\mathrm{SO}_{3}-\mathrm{SiO}_{2} @ \mathrm{DCNPs}$ with a sulfonic silicon dioxide shell coated on the surface of down-conversion nanoparticles (DCNPs) was fabricated by the hydrolysis of 3-mercaptopropyl triethoxysilane (MPTES) and next oxidation. In addition, DCNPs doped with ytterbium ions were synthesized through a feasible two-step method including complexing, coprecipitation, and calcination. The concentration of ytterbium ions and calcination temperature were moreover investigated to optimize the fluorescence feature of DCNPs.

\section{Experimental Section}

2.1. Materials. $\mathrm{YbCl}_{3} \cdot 6 \mathrm{H}_{2} \mathrm{O}(\geq 99.99 \%)$ and $\mathrm{YCl}_{3} \cdot 6 \mathrm{H}_{2} \mathrm{O}$ $(\geq 99.99 \%)$ were purchased from Zhongxin New Materials Co., Ltd. Sodium benzoate (SB, $\geq 99.7 \%), \mathrm{NaF}(\geq 99.7 \%)$, ethanol (EA, $\geq 99.7 \%)$, hydrogen peroxide $\left(\mathrm{H}_{2} \mathrm{O}_{2}, 30 \%\right)$, acetic acid (Ac, 30\%), $\mathrm{NH}_{3} \cdot \mathrm{H}_{2} \mathrm{O}(27 \%), \mathrm{NaOH}(99.7 \%)$, and $\mathrm{HCl}(36 \sim 38 \%)$ were purchased from Sinopharm Chemical Reagent Co., Ltd. 3-Mercaptopropyl triethoxysilane (MPTES, 98\%) was purchased from Shanghai Vita Chemical Reagent Co., Ltd. (Shanghai, China). Pristine down fiber was purchased from Hangzhou Xiaoshan Xintang Feather Co., Ltd. (Hangzhou, China). All the chemicals were used as received without any further purification and ultrapure water was used in all experiments.

2.2. Preparation of Fluorescent DCNPs. In order to improve the conversion yield of products, SB with higher solubility than previous complexants was used as an alternative to synthesize a series of fluorescent DCNPs with various ratios of ytterbium ions and yttrium ions according to the literature [19]. Briefly, a $20 \mathrm{~mL}$ solution with $0.2 \mathrm{M}$ gross cation concentration were prepared by $\mathrm{YbCl}_{3} \cdot 6 \mathrm{H}_{2} \mathrm{O}$ and $\mathrm{YCl}_{3} \cdot 6 \mathrm{H}_{2} \mathrm{O}$ dissolving in deionized water. Then, $0.012 \mathrm{~mol}$ $\mathrm{SB}$ was added into the solution to form the metal-SB complex under vigorous stirring. The complex solution was injected into $60 \mathrm{~mL} 0.83 \mathrm{M} \mathrm{NaF}$ solution rapidly, following was stirred for $1 \mathrm{~h}$. The precipitates of raw nanoparticles were gained by centrifuging and washing twice by deionized water and EA.

Afterwards, the raw nanoparticles were annealed under a nitrogen atmosphere at $400^{\circ} \mathrm{C}$ and maintaining the temperature for $4 \mathrm{~h}$. The resulting DCNPs were collected eventually after cooling down to room temperature.

2.3. Preparation of Anticounterfeiting Particles $\mathrm{SO}_{3}-\mathrm{SiO}_{2} @$ DCNPs. The as-prepared inorganic DCNPs were functionalized with sulfonic groups in two steps. Firstly, $50 \mathrm{~mL}$ EA, $1.5 \mathrm{~mL}$ MPTES, and $0.1 \mathrm{~g}$ DCNPs were mixed by sonication for $30 \mathrm{~min}$ to form a suspension. Then, $0.5 \mathrm{~mL}$ $\mathrm{NH}_{3} \cdot \mathrm{H}_{2} \mathrm{O}$ was added into this mixture quickly followed by stirring for $4 \mathrm{~h}$ to obtain an intermediate product $\left(\mathrm{SH}-\mathrm{SiO}_{2} @\right.$ DCNPs) which is a modification with a sulfhydryl silica shell $[20,21]$. After that, a solution consisting of $5 \mathrm{~mL} \mathrm{H}_{2} \mathrm{O}_{2}$ and $25 \mathrm{~mL}$ Ac was added to oxidate the sulfhydryl group into the sulfonic group. Finally, the sulfonic functionalized core-shell nanoparticles $\mathrm{SO}_{3}-\mathrm{SiO}_{2} @ \mathrm{DCNPs}$ were collected by centrifugation, followed by washing with EA for 4 times and drying.

\subsection{Preparation of Security Down Fiber by Finishing.} Considering the performance decrease contributed by binder, the combination drive between down and mark particles, $\mathrm{SO}_{3}-\mathrm{SiO}_{2} @ \mathrm{DCNPs}$, was inspired by protein fiber coloring, that is, an adhesive-free process. Typically, a mixture of $0.5 \mathrm{~g}$ down fiber, $0.15 \mathrm{~g} \mathrm{SO}_{3}-\mathrm{SiO}_{2} @ \mathrm{DCNPs}$, and $100 \mathrm{~mL}$ deionized water was stirred for $2 \mathrm{~h}$, which was previously adjusted $\mathrm{pH}$ to 3 . Afterwards, the security down fiber was separated by filter, followed by washing with deionized water for 4 times and drying in vacuum at the temperature of $105^{\circ} \mathrm{C}$ for $30 \mathrm{~min}$.

2.5. Measurements and Characterizations. Fluorescence emission spectra of DCNPs, $\mathrm{SO}_{3}-\mathrm{SiO}_{2} @ \mathrm{DCNPs}$, and security down fiber were characterized by Edinburgh Instruments FLS980 fluorescence spectrometers using $980 \mathrm{~nm}$ and $900 \mathrm{~nm}$ lasers (both lasers were purchased from Changchun New Industries Optoelectronics Tech. Co., Ltd.) as excitation light. The Fourier transform infrared (FT-IR) spectra were obtained using Thermo Scientific Nicolet iS10 FT-IR spectrometers ranging from $400 \mathrm{~cm}^{-1}$ to $4000 \mathrm{~cm}^{-1}$. X-ray diffraction (XRD) measurement was recorded on Bruker D2 PHASER diffractometer with $\mathrm{Cu} \mathrm{K} \alpha$ radiation $(40 \mathrm{kV}, 20 \mathrm{~mA})$. X-ray photoelectron spectroscopy (XPS) was carried out using Thermo Fisher Escalab 250Xi with the $\mathrm{Al} \mathrm{K} \alpha$ radiation. The morphology and size distribution of as-prepared products were examined by transmission electron microscopy (TEM) using a JEM-2100 electron microscope operating at $200 \mathrm{kV}$. The surface charges of down fiber previously ground into powder and $\mathrm{SO}_{3}-\mathrm{SiO}_{2} @ \mathrm{DCNPs}$ were studied at different $\mathrm{pH}$ levels by Zeta potential analysis using a Malvern Zetasizer Nano. 


\section{Result and Discussion}

3.1. Morphology and Structure Analysis of DCNPs. The size of as-prepared DCNPs was analyzed by TEM. It can be seen from Figures 1(a) and 1(b) that the particles are almost spherical in shape with a mean diameter of approximately $86 \mathrm{~nm}$. Unlike raw nanoparticles, the DCNPs after annealing were agglomerated, which can be attributable to the calcination atmosphere opposite to previous literature [19]. In terms of the lack of equipment, no further improvement of the preparation was investigated. The crystal phase of DCNPs was further confirmed by X-ray diffraction, as shown in Figure 1(c). Peaks appearing in DCNPs before annealing can be assigned to the cubic phase NaYF4 (JCPDS No. 77-2042), whereas DCNPs tuned to be hexagonal phase (JCPDS 160334) after annealing [22]. Generally, the hexagonal phase $\mathrm{NaYF}_{4}$ crystals are fascinated materials for fluorescence owing to the low phonon energy of the crystal lattice [23]. The diffraction pattern exhibits six peaks at $29.75^{\circ}, 30.64^{\circ}, 39.46^{\circ}, 43.29^{\circ}, 52.90^{\circ}$, and $53.47^{\circ}$, corresponding to (110), (101), (111), (201), (300), and (211) of hexagonal phase $\mathrm{NaYF}_{4}$, respectively.

The DCNPs, as a tracing element in the anticounterfeiting system, are expected to launch infrared fluorescence rather than visible light under being illuminated, thus the optical characteristics of as-prepared DCNPs with different ytterbium doping concentrations were investigated. Figure 1(d) depicts the photoluminescence (PL) spectra of DCNPs excited at $900 \mathrm{~nm}$, indicating that the whole samples of DCNPs emitted a broad downconversion infrared emission corresponding to ${ }^{2} \mathrm{~F}_{5 / 2} \longrightarrow$ ${ }^{2} \mathrm{~F}_{7 / 2}$ transitions of trivalent ytterbium ions. [24] The DCNPs possessed a strongest down-conversion infrared emission when the ytterbium ion doping concentration was $5 \%$. Additionally, the infrared emission was multimodal at possible $976 \mathrm{~nm}, 986 \mathrm{~nm}, 993 \mathrm{~nm}$, and $1025 \mathrm{~nm}$. A popular explanation was that Stark splitting occurred to both ground state and excited state of $\mathrm{Yb}^{3+}$ in glasses, therefore resulting in the multiple energy transitions. that leaded to the multimodal emission [25].

Surprisingly, an apparent green fluorescence was simultaneously observed in some samples and further speculated that was a cooperative up-conversion luminescence. For convenience, a more common $980 \mathrm{~nm}$ laser was applied as an excitation to survey the visible luminescence of DCNPs. Figure 2(a) shows that the intensity of visible luminescence was weakest, which could not be able to be detected by naked eye, when the ytterbium ion doping concentration was $5 \%$. The power dependences of the visible emissions were examined to investigate the luminescence mechanism, as shown in Figure 2(b). The slope of log-log plot of the luminescence intensities as a function of the pump powers was 1.7, which confirmed that the fluorescence mechanism of visible emission was a two-photon process. To obtain strong infrared emission and reduce the intensity of green luminescence, the DCNPs of 5\% ytterbium ion doping concentration were selected as tracing elements in the anticounterfeiting system.
3.2. Morphology and Structure Analysis of $\mathrm{SO}_{3}-\mathrm{SiO}_{2} @ D C N P s$. Although the as-prepared DCNPs were proven to be potential for authentication, the challenge of linkage between DCNPs and down fiber hindered the anticounterfeiting application by the lack of active groups of DCNPs. The modification to DCNPs was implemented to introduce the negative sulfonate groups, as functional groups that forced on the forming of assembly security down fiber, which imparts a driving force, the electrostatic interaction, between DCNPs and the down. Figure 3(a) illustrates the coated materials $\mathrm{SO}_{3}-\mathrm{SiO}_{2} @ \mathrm{DCNPs}$ with a layer of thickness ranging from 10 to $20 \mathrm{~nm}$. Nevertheless, the volume of $\mathrm{SO}_{3}$ $\mathrm{SiO}_{2} @ \mathrm{DCNPs}$ enlarged dramatically due to previous agglomeration of DCNPs.

FT-IR was carried out to characterize the chemical structure of synthetic $\mathrm{SO}_{3}-\mathrm{SiO}_{2} @ \mathrm{DCNPs}$ and the precursor, as shown in Figure 3(b). After the hydrolysis of MPTES, two new presences of peaks at $2556 \mathrm{~cm}^{-1}$ and $2930 \mathrm{~cm}^{-1}$ in SH$\mathrm{SiO}_{2} @ \mathrm{DCNPs}$ can be assigned to the stretching vibration of $\mathrm{S}-\mathrm{H}$ and asymmetric stretching vibration of $-\mathrm{CH}_{2^{-}}$, respectively. By contrast to $\mathrm{SH}-\mathrm{SiO}_{2} @ \mathrm{DCNPs}$, a stronger peak at $3438 \mathrm{~cm}^{-1}$ resulted from the vibration of $\mathrm{O}-\mathrm{H}$ group in $\mathrm{SO}_{3}-\mathrm{SiO}_{2} @ \mathrm{DCNPs}$, which could be contributed to the introduction of $\mathrm{Si}-\mathrm{O}$, a group easy to form hydrogen bonds. Upon treatment with oxidation, the $\mathrm{S}-\mathrm{H}$ stretching vibration disappeared in $\mathrm{SO}_{3}-\mathrm{SiO}_{2} @ \mathrm{DCNPs}$, which could be attributed to the conversion of sulfhydryl group to sulfonic group. Furthermore, a broad peak, being attributed to the stretching vibration of $\mathrm{Si}-\mathrm{O}-\mathrm{Si}$, ranging from $1000 \mathrm{~cm}^{-1}$ to $1350 \mathrm{~cm}^{-1}$ became stronger and wider gradually during the modification process, since it was overlapped with the peak corresponding to the stretching vibration of $\mathrm{S}=\mathrm{O}$, which demonstrated the successful modification of $\mathrm{SO}_{3}-\mathrm{SiO}_{2} @$ DCNPs.

X-ray photoelectron spectroscopy (XPS) was used to analyze the surface of $\mathrm{SO}_{3}-\mathrm{SiO}_{2} @ \mathrm{DCNPs}$ and characterize the sulfur oxidation state. The XPS survey spectra presented several predominant peaks at $1072 \mathrm{eV}, 532 \mathrm{eV}, 685 \mathrm{eV}$, $285 \mathrm{eV}, 159 \mathrm{eV}$, and $103 \mathrm{eV}$, corresponding to $\mathrm{Na} 1 \mathrm{~s}, \mathrm{O} 1 \mathrm{~s}, \mathrm{~F}$ 1s, C 1s, Y 3d, and Si 2p elements, respectively, as shown in Figure 3(c). As shown in Figure 3(d), the peak observed at $169 \mathrm{eV}$ in the $\mathrm{SO}_{3}-\mathrm{SiO}_{2} @ \mathrm{DCNPs}$ could be attributed to the high valent $S 2 p$, indicating the presence of sulfonate group. In addition, no obvious peak at $162 \mathrm{eV}$ of lower valent $S$ was found at such a range of spectra, confirming that the sulfhydryl group was completely tuned to sulfonic group by oxidation $[26,27]$.

The down-conversion infrared fluorescence of $\mathrm{SO}_{3}$ $\mathrm{SiO}_{2} @ \mathrm{DCNPs}$ was investigated, as shown in Figure 4. Upon $900 \mathrm{~nm}$ excitation, the $\mathrm{SO}_{3}-\mathrm{SiO}_{2} @ \mathrm{DCNPs}$ has a similar broad multimodal emission ranging from $940 \mathrm{~nm}$ to $1100 \mathrm{~nm}$ to the DCNPs, which had already been interpreted that the emission was assigned to the ${ }^{2} \mathrm{~F}_{5 / 2} \longrightarrow{ }^{2} \mathrm{~F}_{7 / 2}$ transitions of $\mathrm{Yb}^{3+}$. Since the coating process decreased the surface defects of the DCNPs, the intensity of red-shifted emission became stronger after modification, which is beneficial to anticounterfeiting [28]. Additionally, no visible fluorescence was detected by naked eyes, as the ytterbium doping concentration of $\mathrm{SO}_{3}-\mathrm{SiO}_{2} @ \mathrm{DCNPs}$ was 5\%. 


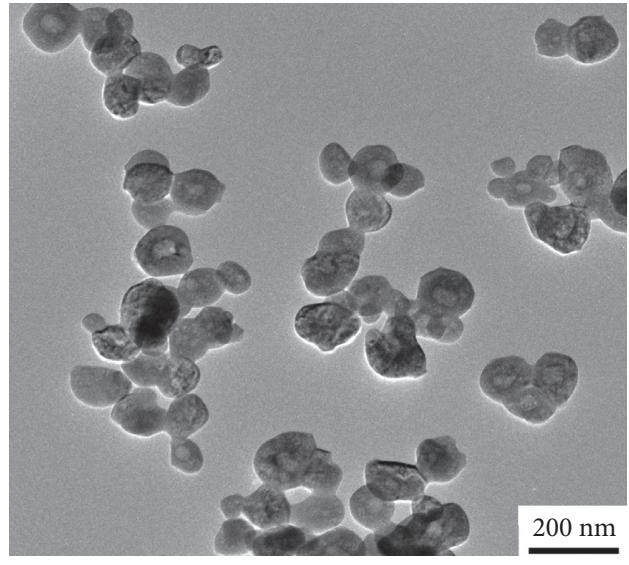

(a)

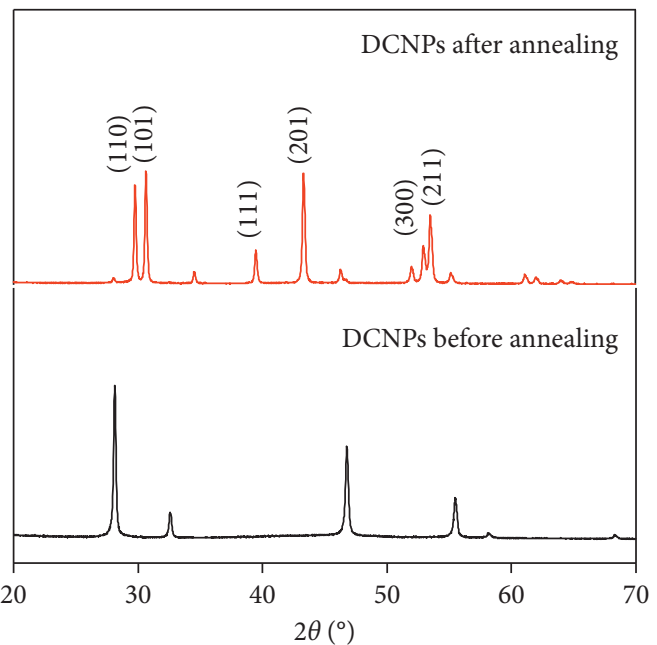

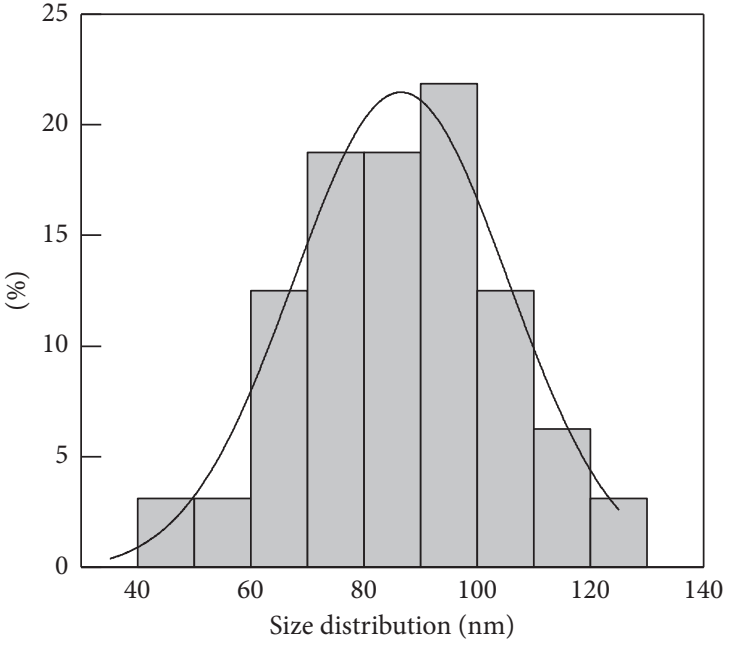

(b)

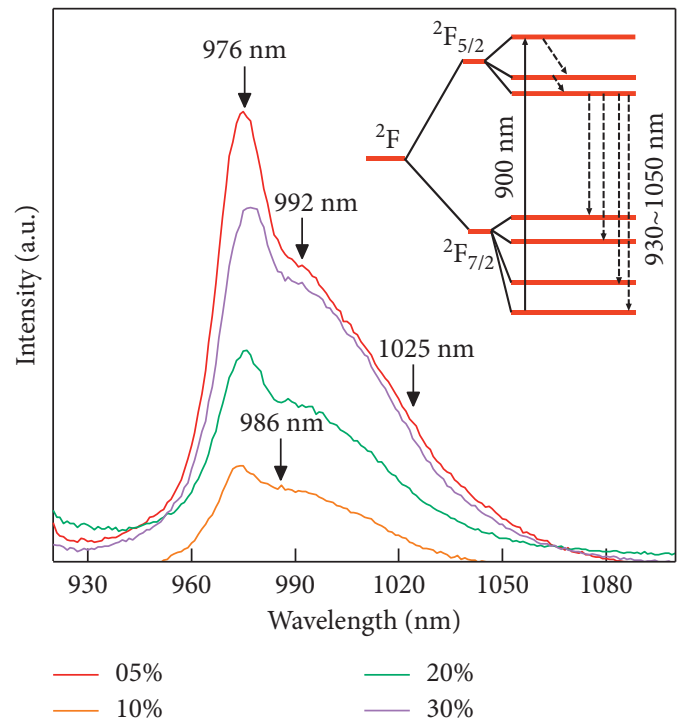

(d)

Figure 1: (a) TEM images of DCNPs. (b) Size distribution of DCNPs. (c) XRD patterns of DCNPs. (d) PL spectra of DCNPs upon 900 nm excitation at various ytterbium doping concentrations, ranging from $5 \%$ to $30 \%$.

3.3. Zeta Potential Examination. As an outstanding filler for thermal garments, down fiber is facing the increasing pressures of counterfeiting. Down-conversion fluorescent materials can be used to mark down fiber to distinguish the certified products among fakes. In this paper, the key of preparation of security down fiber is the rational $\mathrm{pH}$ value, since potential of both down fiber and mark particles are affected $\left(\mathrm{SO}_{3}-\mathrm{SiO}_{2} @ \mathrm{DCNPs}\right)$ by the $\mathrm{pH}$ value of finishing suspension. Figure 5 shows the Zeta potential value of down fiber and mark particles upon different $\mathrm{pH}$ values. The Zeta potential value of down fiber and mark particles declined with the increase of $\mathrm{pH}$ value, and the potential of mark particles remained negative. Nevertheless, an obvious transition at $\mathrm{pH}$ of 3 to 4 could be observed in the plot of down fiber, in which the potential of down fiber beginning tuned from positive to negative. According to this result, the $\mathrm{pH}$ of 3 was selected as a parameter of finishing suspension.
3.4. Morphology and Optical Property Analysis of Security Down Fiber. The morphologies of the security down fiber were captured by scanning electron microscopic (SEM) images, as shown in Figure 6. Figure 6(a) shows that numerous microscale mark particles attached to the surface of fiber at random or lived in the bifurcation after finishing, indicating that the mark particles were successfully loaded on the fiber. To investigate the combination between down fiber and mark particles, the washing process of security down fiber against repeated launderings was implemented according to GB/T 8629-2001 standard method. Figure 6(b) displays the morphology of down fiber after washing six times (once experimental washing equal to five times home washing). After washing, the mark particles were almost invisible, but it was found stored in fiber bifurcation under a smaller microscope, which demonstrated that the combination between down fiber and mark particles was valid. 


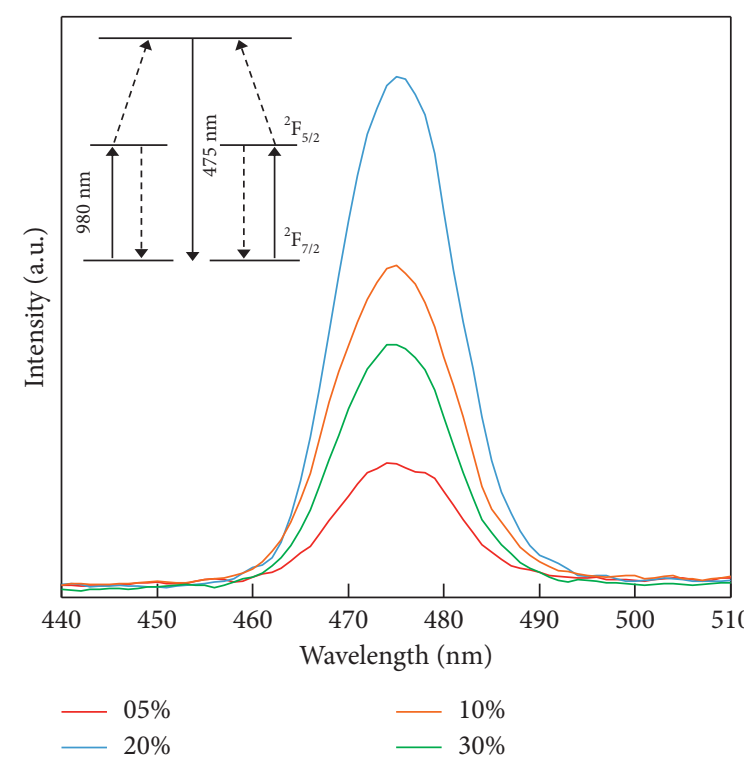

(a)

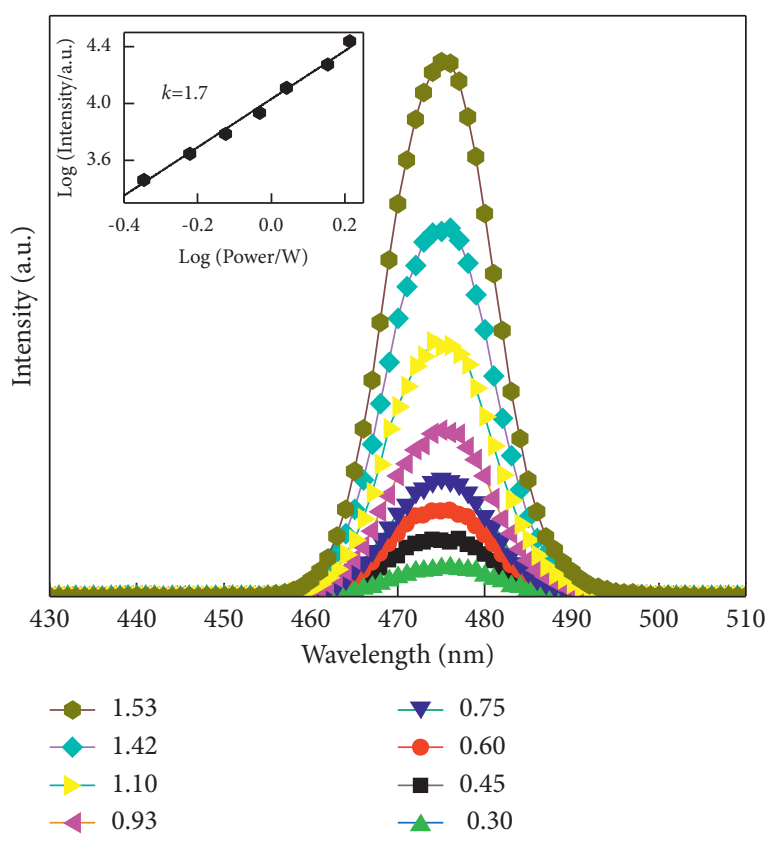

(b)

Figure 2: (a) PL spectra of DCNPs upon $980 \mathrm{~nm}$ excitation at various ytterbium doping concentrations, ranging from $5 \%$ to $30 \%$, and inset in the left is a sketch map of cooperative luminescence for $\mathrm{Yb}^{3+}-\mathrm{Yb}^{3+}$ pair. (b) PL spectra of DCNPs with different excitation powers, ranging from $0.3 \mathrm{~W}$ to $1.53 \mathrm{~W}$, and inset in the left is the log-log plot of the cooperative luminescence intensities as a function of the pump powers.
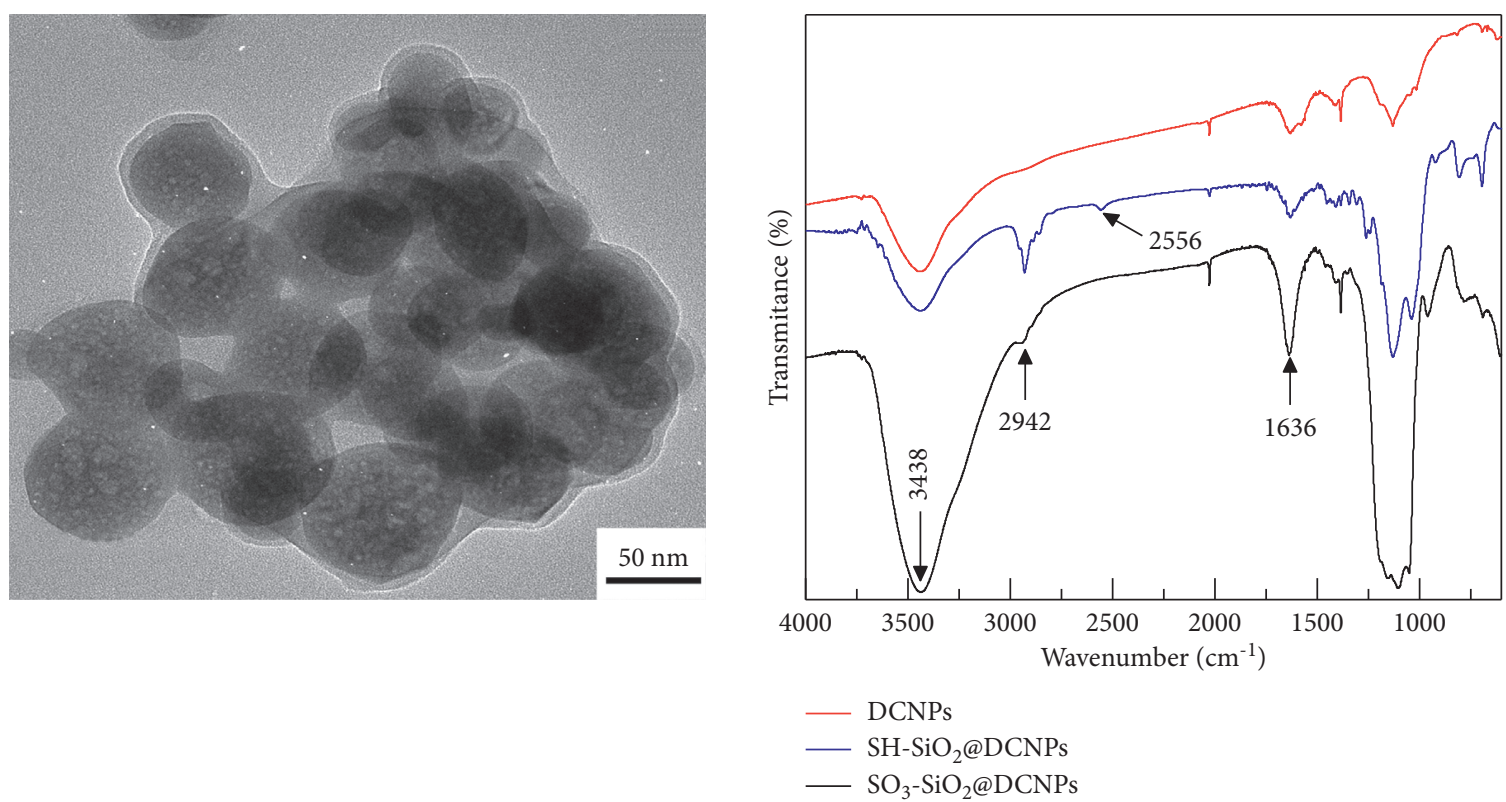

(a)

(b)

FIgURE 3: Continued. 


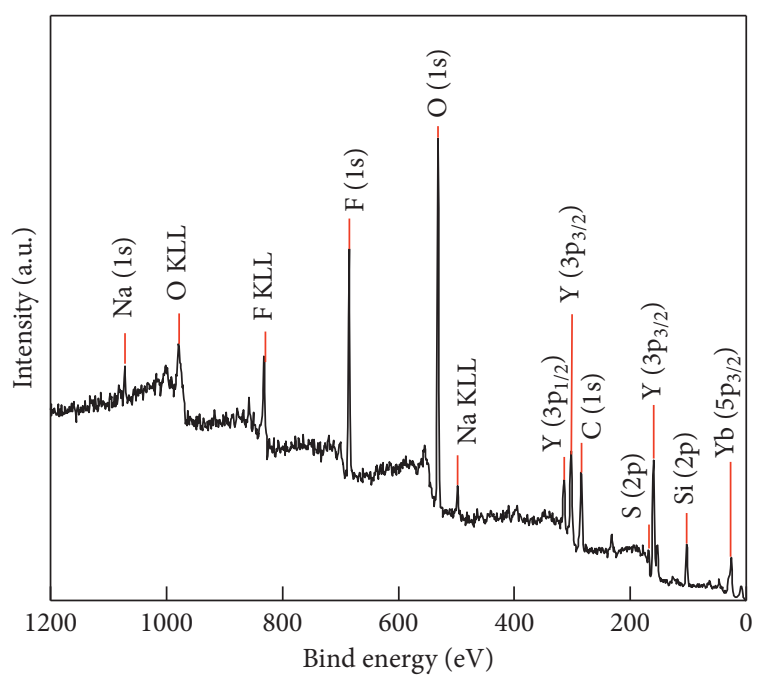

(c)

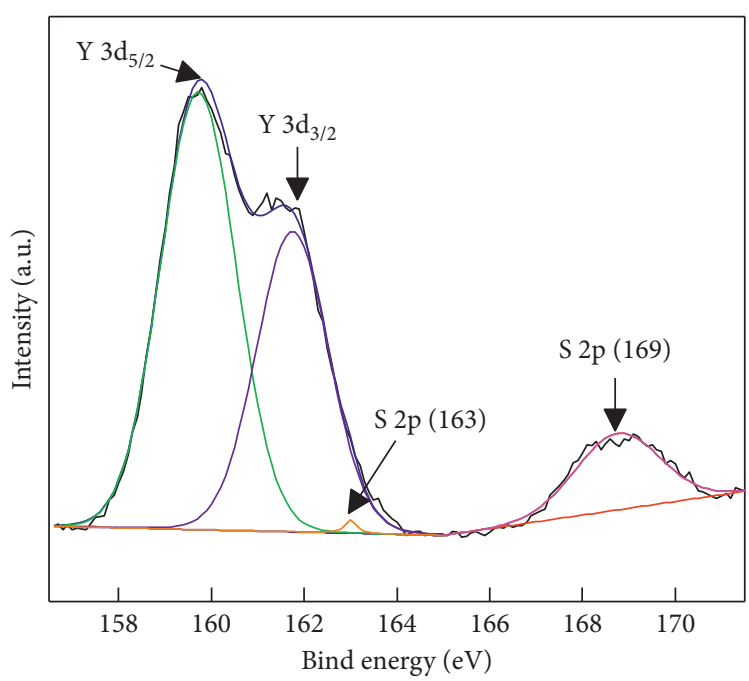

(d)

FIGURE 3: (a) TEM images of $\mathrm{SO}_{3}-\mathrm{SiO}_{2} @ D C N P s$. (b) FT-IR spectrums of DCNPS and modified nanoparticles. (c) XPS survey spectra of $\mathrm{SO}_{3}{ }^{-}$ $\mathrm{SiO}_{2} @$ DCNPs. (d) Deconvolution of S 2p peak of the $\mathrm{SO}_{3}-\mathrm{SiO}_{2} @ \mathrm{DCNPs}$.

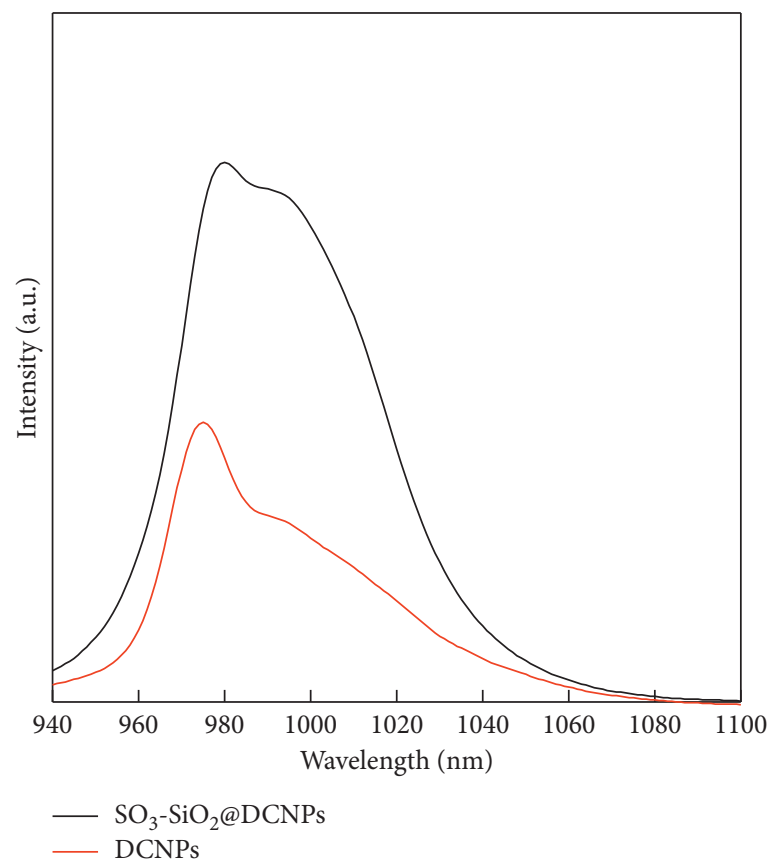

Figure 4: PL spectra of $\mathrm{SO}_{3}-\mathrm{SiO}_{2} @ \mathrm{DCNPs}$ upon $900 \mathrm{~nm}$ excitation at ytterbium doping concentration of 5\%.

Further fluorescence performance of security down fiber was studied by PL spectra, as shown in Figure 7. An infrared emission distinguished from the excitation was emerged, when the security down fiber was illuminated by $900 \mathrm{~nm}$. The broad emission with a Stokes shift of approximately $90 \mathrm{~nm}$ was assigned to the ${ }^{2} \mathrm{~F}_{5 / 2} \longrightarrow{ }^{2} \mathrm{~F}_{7 / 2}$ transitions of $\mathrm{Yb}^{3+}$, and the multimodal feature of the emission was contributed to the Stark splitting of $\mathrm{Yb}^{3+}$ in crystal. The security down fiber after washing had a comparable luminescent property, yet the intensity of emission became weaker due to the decrease in total loading amount of mark particles on fiber. The mark particles, $\mathrm{SO}_{3}-\mathrm{SiO}_{2} @ \mathrm{DCNPs}$, are promising for commercial anticounterfeiting of down fiber owing to its secret infrared emission and easy preparation. 


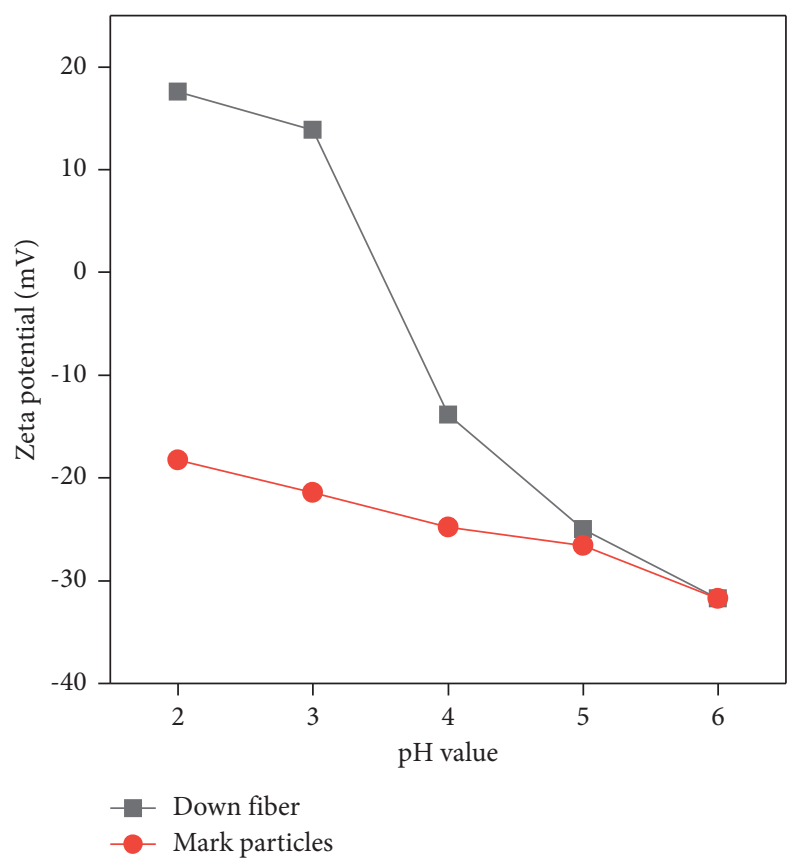

Figure 5: Zeta potential of down fiber and mark particles.
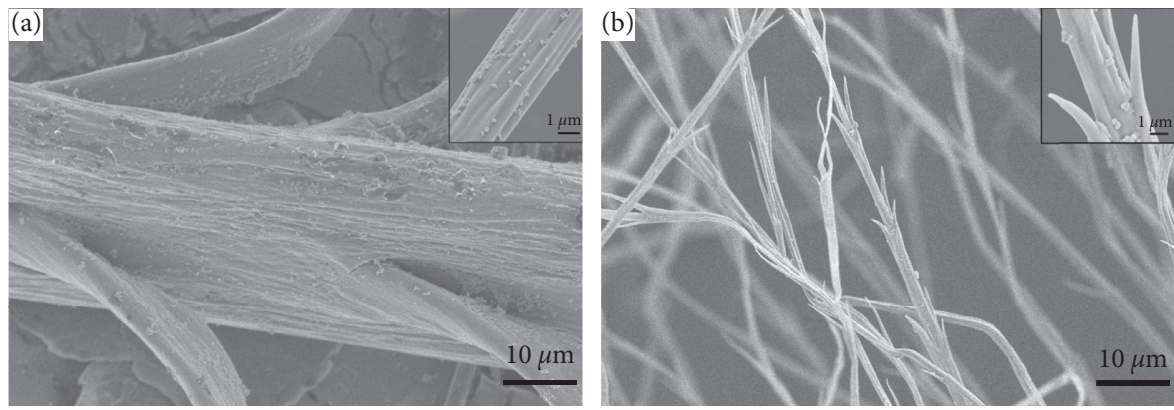

FIgURE 6: SEM pictures of down fiber. (a) Before washing. (b) After washing six times.

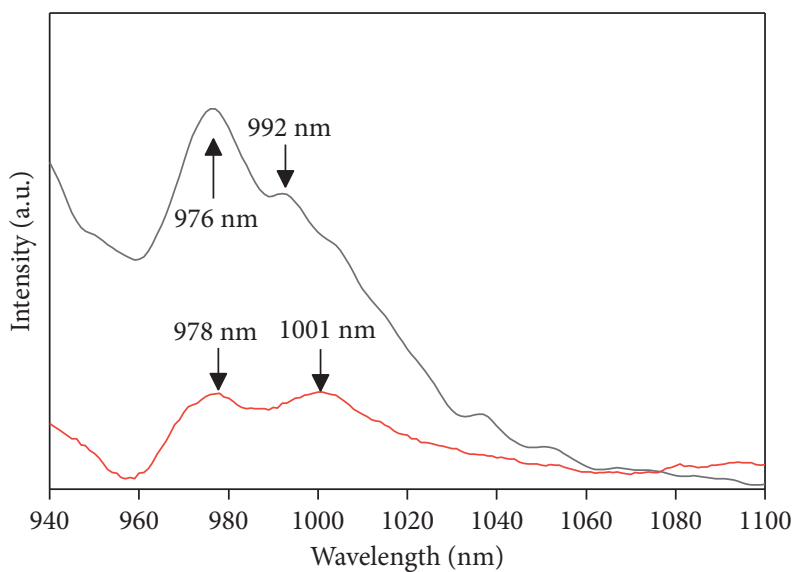

- Security down fiber

— Security down fiber after washing

FIgURE 7: PL spectra of security down fiber upon $900 \mathrm{~nm}$ excitation. 


\section{Conclusions}

The preparation of DCNPs with characteristic fluorescence and its further application on the anticounterfeiting of down fiber were reported in this paper. The fluorescence for anticounterfeiting is a red-shifted emission approximately ranging from $940 \mathrm{~nm}$ to $1100 \mathrm{~nm}$, corresponding to ${ }^{2} \mathrm{~F}_{5 / 2} \longrightarrow{ }^{2} \mathrm{~F}_{7 / 2}$ transitions of $\mathrm{Yb}^{3+}$ in hexagonal $\mathrm{NaYF}_{4}$. These processes were carried out in a solution without any extreme conditions, which is friendly to pragmatic application. As expected, the security down fiber had a comparable down-conversion infrared emission to DCNPs at $900 \mathrm{~nm}$ excitation, and the visible fluorescence was weakened by regulating the ytterbium doping concentration to $5 \%$. In addition, the red-shifted emission of security down fiber did not disappear after washing according to GB/T 8629-2001 standard method, indicating that the combination between down fiber and modified DCNPs is feasible. This strategy, of infrared fluorescence and no adhesive, may open up new possibilities for the anticounterfeiting of superior protein fiber, such as silk, cashmere, and vicuna.

\section{Data Availability}

The figure data and others used to support the findings of this study are included within the article.

\section{Conflicts of Interest}

The authors declare that they have no conflicts of interest.

\section{Acknowledgments}

The authors thank the financial support from the National Key R\&D Program of China (2018YFC0810300) and the Key Laboratory of Eco-Textiles, Ministry of Education (supported by the Fundamental Research Funds for the Central Universities, No. JUSRP52007A).

\section{References}

[1] X. Bian, K.-Y. Wang, A. Smith, and N. Yannopoulou, "New insights into unethical counterfeit consumption," Journal of Business Research, vol. 69, no. 10, pp. 4249-4258, 2016.

[2] T. K. Agrawal, L. Koehl, and C. Campagne, "A secured tag for implementation of traceability in textile and clothing supply chain," International Journal of Advanced Manufacturing Technology, vol. 99, no. 9-12, pp. 2563-2577, 2018.

[3] K. Baatout, F. Saad, A. Baffoun et al., "Luminescent cotton fibers coated with fluorescein dye for anti-counterfeiting applications," Materials Chemistry and Physics, vol. 234, pp. 304-310, 2019.

[4] Z. Bao, C. Lu, C. Qin et al., "Preparation of fluorescent polyacrylonitrile nanofiber membrane based on polymerizable 1,8-naphthalimide fluorescent disperse dye," Fibers and Polymers, vol. 18, no. 6, pp. 1017-1024, 2017.

[5] M. Naftaly, J. F. Molloy, G. V. Lanskii, K. A. Kokh, and Y. M. Andreev, "Terahertz time-domain spectroscopy for textile identification," Applied Optics, vol. 52, no. 19, p. 4433, 2013.
[6] C. Tan, H. Chen, Z. Lin, and T. Wu, "Category identification of textile fibers based on near-infrared spectroscopy combined with data description algorithms," Vibrational Spectroscopy, vol. 100, pp. 71-78, 2019.

[7] W. Wei, D. Deng, L. Zeng, C. Zhang, and W. Shi, "Classification of foreign fibers using deep learning and its implementation on embedded system," International Journal of Advanced Robotic Systems, vol. 16, 2019.

[8] S. Peng, J. Wen, M. Hai et al., "Synthesis and application of reversible fluorescent photochromic molecules based on tetraphenylethylene and photochromic groups," New Journal of Chemistry, vol. 43, no. 2, pp. 617-621, 2019.

[9] Z. Fatahi, N. Esfandiari, and Z. Ranjbar, "A new anti-counterfeiting feature relying on invisible non-toxic fluorescent carbon dots," Journal of Analysis and Testing, vol. 4, no. 4, pp. 307-315, 2020.

[10] M. Skwierczynska, P. Wozny, M. Runowski, M. Perzanowski, P. Kulpinski, and S. Lis, "Bifunctional magnetic-upconverting luminescent cellulose fibers for anticounterfeiting purposes," Journal of Alloys and Compounds, vol. 829, 2020.

[11] S. Xie, G. Gong, Y. Song et al., "Design of novel lanthanidedoped core-shell nanocrystals with dual up-conversion and down-conversion luminescence for anti-counterfeiting printing," Dalton Transactions, vol. 48, no. 20, pp. 6971-6983, 2019.

[12] H. Al Sabea, L. Norel, O. Galangau et al., "Efficient photomodulation of visible $\mathrm{Eu}$ (III) and invisible $\mathrm{Yb}$ (III) luminescences using DTE photochromic ligands for optical encryption," Advanced Functional Materials, vol. 30, 2020.

[13] X. Zhou, L. Z. Fang, S. W. Huang et al., Ultraviolet and nearinfrared luminescence of $\mathrm{Ce}^{3+} / \mathrm{Yb}^{3+}$ co-doping LiLuF4 single crystal, Journal of Inorganic Materials, vol. 35, p. 556, 2020.

[14] Z. Zhang, H. Chang, B. Xue et al., "Near-infrared and visible dual emissive transparent nanopaper based on $\mathrm{Yb}$ (III)-carbon quantum dots grafted oxidized nanofibrillated cellulose for anti-counterfeiting applications," Cellulose, vol. 25, no. 1, pp. 377-389, 2018.

[15] A. Szczeszak, M. Skwierczyńska, D. Przybylska et al., "Upconversion luminescence in cellulose composites (fibres and paper) modified with lanthanide-doped SrF2 nanoparticles," Journal of Materials Chemistry C, vol. 8, no. 34, Article ID 11922, 2020.

[16] J. X. Xu, G. Du, C. Tong et al., "Controlled synthesis and panchromatic printing of highly luminescent NaYF4: $\mathrm{Ln}^{3+}$ upconversion hollow microtubes for information encryption on various packaging substrates," Journal of Photochemistry and Photobiology A-Chemistry, vol. 396, 2020.

[17] Z. Pang, Y. Zhu, and M. Ge, "Study on the structural and luminous properties of two kinds self-luminous fibers," Journal of the Textile Institute, vol. 111, no. 3, pp. 345-351, 2020.

[18] I. S. Marae, W. Sharmoukh, E. A. Bakhite, O. S. Moustafa, M. S. Abbady, and H. E. Emam, "Durable fluorescent cotton textile by immobilization of unique tetrahydrothienoisoquinoline derivatives," Cellulose, vol. 28, no. 9, 2021.

[19] G. Yi, H. Lu, S. Zhao et al., "Synthesis, characterization, and biological application of size-controlled $\mathrm{n}$ infrared-to-visible up-conversion phosphors," Nano Letters, vol. 4, no. 11, pp. 2191-2196, 2004.

[20] T.-J. Lee, L.-K. Chau, and C.-J. Huang, "Controlled s: high molecular regularity of functional thiol groups on siloxane coatings," Langmuir, vol. 36, no. 21, pp. 5935-5943, 2020. 
[21] G. Mondin, M. R. Lohe, F. M. Wisser et al., "Electroless copper deposition on (3-mercaptopropyl)triethoxysilane-coated silica and alumina nanoparticles," Electrochimica Acta, vol. 114, pp. 521-526, 2013.

[22] S. Saha, R. G. S. Pala, and S. Sivakumar, "Catalyzing cubic-tohexagonal phase transition in NaYF4 via ligand enhanced surface ordering," Crystal Growth \& Design, vol. 18, no. 9, pp. 5080-5088, 2018.

[23] Y. Hu, Q. Shao, P. Zhang, Y. Dong, F. Fang, and J. Jiang, "Mechanistic investigations on the dramatic thermally induced luminescence enhancement in upconversion nanocrystals," Journal of Physical Chemistry C, vol. 122, no. 45, Article ID 26142, 2018.

[24] G. Zhang, I. Milisavljevic, K. Grzeszkiewicz, P. Stachowiak, D. Hreniak, and Y. Wu, "New optical ceramics: high-entropy sesquioxide $\mathrm{X}_{2} \mathrm{O}_{3}$ multi-wavelength emission phosphor transparent ceramics," Journal of the European Ceramic Society, vol. 41, no. 6, pp. 3621-3628, 2021.

[25] B. Yang, X. Liu, X. Wang, J. Zhang, L. Hu, and L. Zhang, "Compositional dependence of room-temperature Stark splitting of $\mathrm{Yb}^{3+}$ in several popular glass systems," Optics Letters, vol. 39, no. 7, p. 1772, 2014

[26] P.-P. Lu, Z.-L. Xu, H. Yang, and Y.-M. Wei, "Processingstructure-property correlations of $\mathrm{pp}$ acid nanofibers fabricated via electrospinning from polymer-nanoparticle suspensions," ACS Applied Materials \& Interfaces, vol. 4, no. 3, pp. 1716-1723, 2012.

[27] X. Rao, M. Tatoulian, C. Guyon, S. Ognier, C. Chu, and A. Abou Hassan, "A comparison study of functional groups (amine vs. Thiol) for immobilizing AuNPs on zeolite surface," Nanomaterials, vol. 9, 2019.

[28] M. Mondal, V. K. Rai, and C. Srivastava, "Influence of silica surface coating on optical properties of $\mathrm{Er}^{3+}-\mathrm{Yb}^{3+}$ : $\mathrm{YMoO} 4$ upconverting nanoparticles," Chemical Engineering Journal, vol. 327, pp. 838-848, 2017. 